\title{
Conceptualizing, Contextualizing, and Operationalizing Race in Quantitative Health Sciences Research
}

\author{
Elle Lett, MBiostat, MA, $P b D^{1-4}$ \\ Emmanuella Asabor, MPbil ${ }^{1,5,6}$ \\ Sourik Beltrán, $M D, M B E^{7}$ \\ Asbley Micbelle Cannon, $B^{8}$ \\ Onyebucbi A. Arab, MD, PbD $D^{9-11}$ \\ ${ }^{1}$ Center for Health Equity Advancement, \\ University of Pennsylvania, Philadelphia, \\ Pennsylvania
}

${ }^{2}$ Center for Applied Transgender Studies, Chicago, Illinois

${ }^{3}$ Perelman School of Medicine, University of Pennsylvania, Philadelphia, Pennsylvania

${ }^{4}$ Palliative and Advanced Illness Research (PAIR) Center, University of Pennsylvania, Philadelphia, Pennsylvania

${ }^{5}$ Yale University School of Medicine, New Haven, Connecticut

${ }^{6}$ Department of Epidemiology of Microbial Diseases, Yale University School of Public Health, New Haven, Connecticut

${ }^{7}$ Department of Medicine, Massachusetts General Hospital, Boston, Massachusetts

${ }^{8}$ Technical Resources International, Bethesda, Maryland

${ }^{9}$ Department of Epidemiology, Fielding School of Public Health, University of California, Los Angeles, Los Angeles, California

${ }^{10}$ Department of Statistics, University of California, Los Angeles College of Letters and Science, Los Angeles, California

"Department of Public Health, Aarhus University, Aarhus, Denmark

Conflicts of interest: authors report none.

\section{CORRESPONDING AUTHOR}

Elle Lett

University of Pennsylvania

Blockley Hall

Philadelphia, PA 19146

ellel@upenn.edu

\begin{abstract}
Differences in health outcomes across racial groups are among the most commonly reported findings in health disparities research. Often, these studies do not explicitly connect observed disparities to mechanisms of systemic racism that drive adverse health outcomes among racialized and other marginalized groups in the United States. Without this connection, investigators inadvertently support harmful narratives of biologic essentialism or cultural inferiority that pathologize racial identities and inhibit health equity. This paper outlines pitfalls in the conceptualization, contextualization, and operationalization of race in quantitative population health research and provides recommendations on how to appropriately engage in scientific inquiry aimed at understanding racial health inequities. Race should not be used as a measure of biologic difference, but rather as a proxy for exposure to systemic racism. Future studies should go beyond this proxy use and directly measure racism and its health impacts.
\end{abstract}

\section{VISUAL ABSTRACT}

Ann Fam Med 2022;20:Online. https://doi.org/10.1370/afm.2792.

Annals "Online First" article. Accepted for publication in a later issue.

\section{INTRODUCTION}

$\mathrm{T}$ The racialized health impacts of police violence ${ }^{1}$ and the COVID-19 pandemic $^{2}$ have brought necessary conversations on racial injustice to the foreground of scientific and political discourse in the United States. Scientific and medical communities have been responsive with researchers increasingly studying racial health inequities. Academic publications have appropriately followed suit with special editions from journals such as JAMA, Health Affairs, and the American Journal of Public Health. Despite this, many historic and contemporary works documenting racial health disparities frequently recapitulate issues in the conceptualization, contextualization, and operationalization of race in quantitative health sciences research. Here we outline some of these specific pitfalls, discuss their implications, and provide recommendations for researchers to begin to address them.

The scientific record is saturated with research demonstrating differences in health outcomes between racial and ethnic groups. Where these studies often fall short is in linking racial health disparities to the precise mechanisms that produce them such as manifestations of racism including poverty and state violence. ${ }^{3-5}$ As a result, alternative explanations including biologic essentialism ${ }^{6}$ or cultural inferiority ${ }^{7}$ may arise to explain observed differences in health outcomes, which can further pathologize individuals having these disparities. This has been seen throughout the COVID-19 pandemic where inequities in morbidity and mortality among Black individuals have been incorrectly attributed to harmful stereotypes such as genetic susceptibility, ${ }^{8,9}$ greater risk tolerance or willful assumption of pandemic risk ${ }^{10}$ or worse hygiene ${ }^{11}$ despite an absence of supporting evidence. To avoid these issues, we suggest understanding race as a proxy measure for exposure to experiences of structural and individual racism. ${ }^{12} \mathrm{It}$ is through this conceptualization that racial disparity, or difference in health outcomes across racialized groups, can be understood as racial inequity, a lack of health justice.

\section{WHAT IS RACE?}

Race and ethnicity are categories that signify both social meaning (ie, social construction of race) as well as material stratification (eg, observed socioeconomic 
differences across race).$^{13}$ Before modern understandings of race, Western scholars historically constructed race as purely biologic, extrapolating that phenotypic similarity could determine things like behavior, intellect, and morality. ${ }^{14}$ While modern scholars may not explicitly traffic in this savage-civilized dichotomy, many contemporaries continue to "biologitisize" race $^{15-17}$ despite overwhelming evidence disputing the biologic meaning or usefulness of race..$^{18,19}$

While race is known to be a poor proxy for biologic or genetic difference, it remains salient because of how it impacts social relations. ${ }^{20}$ Racialization is the process through which social meaning is assigned to individuals or groups based on shared characteristics such as phenotype, culture, language, nationality, religion, and class. ${ }^{20}$ In the United States, racialization leads individuals to be grouped based on these characteristics, thus giving rise to the idea of "minority groups." Racialized individuals are then said to have been "minoritized" (ie, to have been made to be a minority) a term which emphasizes the active, and therefore reversible, status of their racialization. In a critical race theory framework, White supremacy is a fundamental component of American society. ${ }^{21}$ Thus, because racism is ordinary and ubiquitous, race takes on value as a proxy for shared experience (rather than biology). For marginalized groups, one dimension of this experience is shared oppression. In other words, while racial categories are not biologically meaningful, they have become an indelible marker for overlapping experiences of racialization as well as the historical, political, and social processes which shape our daily lives. Unfortunately, health disparities research often continues to suggest biologic essentialism as the mechanism behind inequitable health outcomes. We emphasize that an understanding of race that moves from physiologic difference to a particular relationship to structural forces is foundational to high quality health equity research.

\section{Conceptualization: Race as a Proxy for Exposure to Racism}

One of the most challenging aspects of the persistent failure to properly study racial and ethnic health is that theoretical resources to aid the process are extensive, including fundamental cause theory, ${ }_{1}^{22}$ ecosocial theory, ${ }_{1}^{23-25}$ and the Public Health Critical Race Praxis. ${ }^{26}$ In fundamental cause theory, disparities in social conditions associated with race, like income or housing, are understood as the origin of disease because they limit access to health-sustaining resources. ${ }^{22}$ In ecosocial theory, forms of discrimination such as racism are understood as societal forces that become biologically embodied through pathways such as social and economic deprivation, thereby causing disease. ${ }^{25}$ In the Public Health Critical Race Praxis, racism's role in the production of social hierarchy is central to health inequity and, therefore, research that does not challenge such hierarchies is seen as counterproductive. ${ }^{26}$ While each theory carries different assumptions and therefore should be selected to match the specific research question, they all conceptualize race as a proxy for social stratification achieved through systemic racism and resulting in the inequitable distribution of resources that causes negative health consequences for individuals from racialized groups.

There is no consensus definition of systemic racism, but most articulations emphasize that (1) racism is pervasive and can manifest across all societal domains, ${ }^{27-29}$ (2) it is historical with intergenerational effects, ${ }^{29,30}$ and (3) it is driven by White supremacy. ${ }^{27,29}$ Some writers treat systemic racism as synonymous with structural racism. Here we draw a distinction. Systemic racism encapsulates all manifestations of racism which may then be further subdivided to individualized and structural racism. Structural racism therefore refers to the compounding impacts of the cultural norms, policies, laws, and practices that produce racial inequity whereas individualized racism refers to the individual and interpersonal manifestations of racial discrimination (Figure 1).

Among individualized forms of systemic racism, internalized racism involves negative self-perceptions as a result of dominant cultural attitudes of racial inferiority. Internalized racism has been linked to depressive symptoms among Black Americans. ${ }^{31}$ Interpersonal racism is race-based discrimination between individuals such as implicit bias or overt racial discrimination. In health care, this may include false assumptions about BlackWhite physiologic differences in pain tolerance ${ }^{32}$ and therefore underprescribing pain medications to Black patients. ${ }^{33}$

Here, we define institutional racism, or policies and practices within or across institutions of a society that generate racial inequity, ${ }^{34}$ as a subcomponent of structural racism. ${ }^{27} \mathrm{We}$ conceptualize the primary distinction between structural and institutional racism as scope and temporality-structural racism is a characteristic of an entire society (scope) and includes the downstream impacts of historical policies (temporality) such as redlining or disparate sentencing for crack and powder cocaine. ${ }^{35}$ Structural racism also includes intergenerational effects such as epigenetic modifications from preconception or in utero trauma ${ }^{36}$ as well as historical economic deprivation. In contrast, institutional racism might refer to the practices of a single entity or organization within a society, such as an individual school, company, or health system. Both structural and institutional racism can manifest on different scales including national-, state-, and community-levels (counties, neighborhoods, blocks). The boundaries between these are not rigid, and Figure 1 shows our decomposition of the elements of systemic racism. Individual-level racism is influenced by cultural norms that are established as a result of structural racism at a societal level. Structural and institutional racism at different levels feed back and influence one another (Figure 1). This network complexity mirrors reality: systemic racism is a core component of American society and manifests across all its domains.

\section{Contextualization: Identifying Intervention Targets}

Conceptualizing race as a proxy for systemic racism is necessary but not sufficient to ensure high-quality research that 


\section{Figure 1. Elements of systemic racism.}

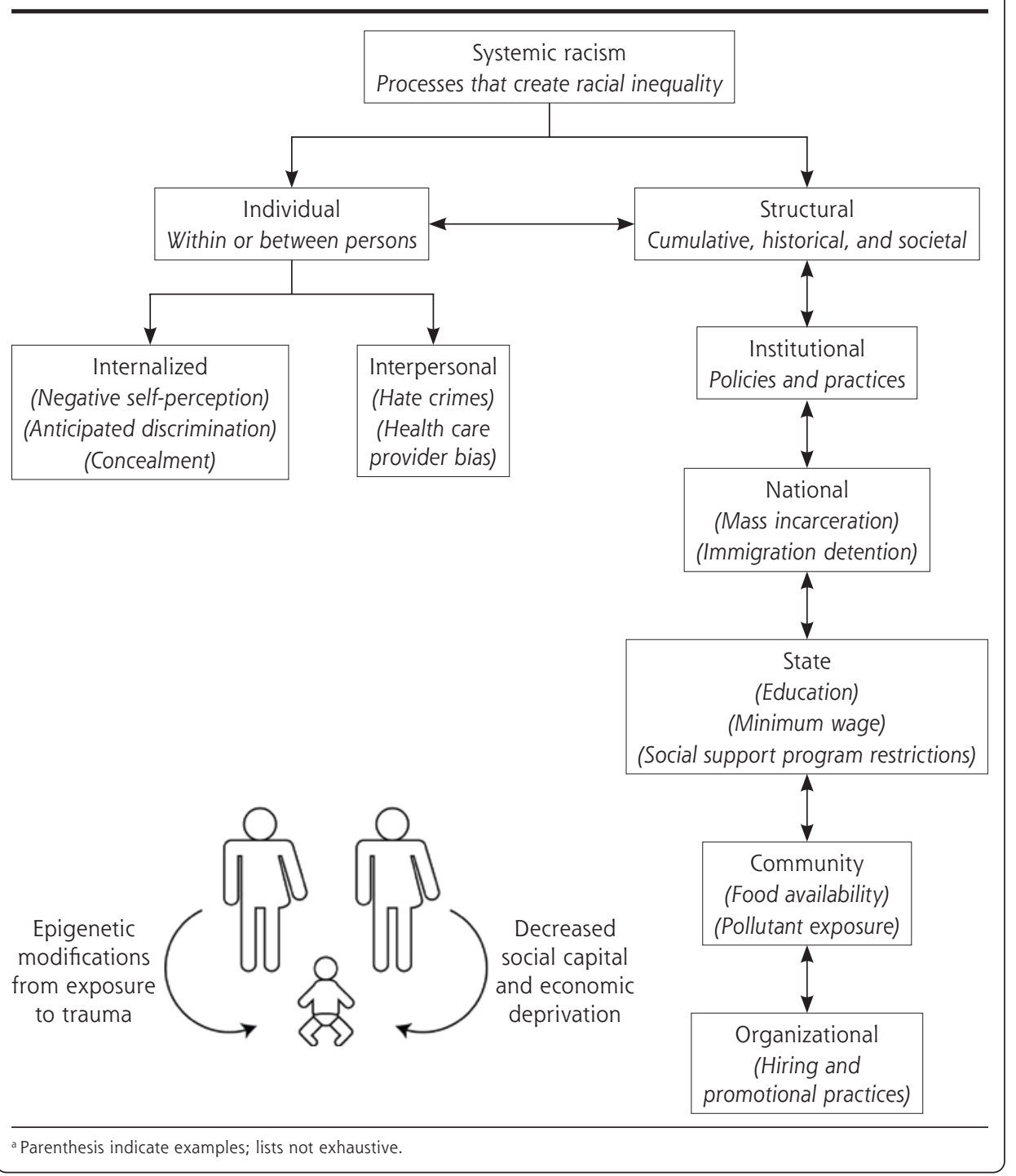

health insurance, level of education, or annual income. However, if race is conceptualized as a proxy for systemic racism, these individuallevel factors are mediators of the relationship between the exposure to systemic racism and health. In this view, factors such as health insurance or level of education serve as points of intervention. Therefore, the additional effect of race that is not accounted for by chosen study measures, is attributable to other unmeasured elements of systemic racism. To that end, more attention should be paid to contextual factors, meso to macro forces that both constitute and are reconstituted by systemic racism, such as residential segregation, food availability, ${ }^{37,38}$ and built neighborhood environment including green space ${ }^{39}$ or proximity to pollutant reservoirs.

Additionally, it is important to note that systemic racism is not experienced in isolation from other forms of marginalization. Intersectionality is a theoretical framework that is increasingly being recognized as an important tool for health equity research. ${ }^{28,40}$ Intersectionality explains how individuals with multiple marginalized identities are subject to interactive mechanisms of oppression. ${ }^{41,42}$ In this framework, systems of oppression are understood to be interactive and mutually constitutive advances racial health equity. By definition, systemic racism is ubiquitous and negatively impacts the health of Black, Latine, Indigneous, and other racialized groups through multiple pathways. Bailey et al enumerated some of these pathways, including state-sanctioned violence (police brutality and incarceration), economic deprivation, and environmental injustice, among others. ${ }^{27}$ Because of the numerous ways that racism drives adverse health outcomes, documenting racial inequity itself serves as a preliminary step in identifying populations in need of support. Then, situating inequity in the context of the specific pathways that produce disparate health outcomes is needed to identify context-specific interventions.

It is common practice for quantitative studies to document conditionally independent associations between race and health outcomes as evidence of racial health inequities. In such work, race is often treated as a nonmodifiable factor that has an additional adverse effect on a health outcome after adjusting for other individual-level factors such as such that a racialized experience is a gendered experience, is also a classed experience, etc. Several transgender population health studies have applied this framework to provide insights to health for multiply marginalized subpopulations (ie, groups that experience multiple forms of marginalization concurrently), ${ }^{43-45}$ and there is a robust literature on methodological approaches for applying this framework. ${ }^{46-48}$

As we reorient ourselves toward estimating the health impacts of systemic racism and other systems of oppression, it becomes difficult to identify appropriate methods for effect quantification. There is active debate between causal inference and social epidemiology about how to improve the estimates of causal effects of social-structural forces such as structural racism. ${ }^{49,50}$ Agent-based models (ABMs) may be a more appropriate approach than traditional causal inference methods because of their flexibility and potential to adapt complex realworld relationships with high fidelity. ${ }^{49,51}$ Rather than having restrictive models of narrow interventions that only impact 
1 domain downstream of systemic racism (ie, estimating the causal effect of providing direct economic aid or stable housing), ABMs allow the investigator to "imagine" and simulate a world where the multitude of interconnected pathways are perturbed concurrently (ie, simulating the positive intergenerational health impacts of removing multiple components of systemic racism such as income inequity or food insecurity), and where interdependence between individuals (ie, social capital) affect the outcome. ${ }^{52,53}$ Other approaches are still being developed and this remains a topic of active investigation. ${ }^{51}$

\section{Operationalization: Measuring Race and Racism}

How investigators operationalize race is a combination of the study question and the available data. Depending on how the "race variable" is constructed, it can be used as a proxy for different aspects of systemic racism. For instance, in survey settings, race is normally self-reported, whereas in studies based on electronic health records, an individual's race may be assigned by a health care worker. Neither of these are more or less correct. Rather, they represent different outputs of the same racialization process. While these measures may be similar for an individual, prior work has demonstrated that there can be considerable discordance. ${ }^{54,55}$ Self-reported race reflects an individual's understanding of their own racial identity. Therefore, using self-reported race encapsulates the internalized racism component of systemic racism with high fidelity. Experiences of interpersonal racism, however, are more directly related to how members of a society interact with an individual's race. In instances of discordance between self-reported and assigned race (eg, a person who self-identifies as multiracial but is primarily perceived to be White), it is possible that internalized racism and interpersonal racism may have distinct or even opposing health impacts. Similarly, because of the intergenerational impact of structural racism, "familial" race may indicate shared exposure to racism over time, which may manifest distinctly from interpersonal and internalized racism depending on individual race. These delineations are not discrete: the greater the concordance between self-reported, assigned, and "familial" race, the more consistent they are as proxy measures for systemic racism. To make the best use of racial identity as a proxy for exposure to the different elements of systemic racism, it is most appropriate to obtain information about self-reported race, how individuals are perceived or their race is assigned, and their familial racial history. Often, investigators will obtain some aspect of assigned and/or familial race, but best practices for acquiring or using this information have yet to be developed.

The last element to consider with operationalization is interrogating whether race categories meaningfully group individuals by shared experiences with systemic racism. Perhaps the most notable example of this is how the "Hispanic" or "Hispanic/Latine" ethnic group is frequently used in health equity literature. "Hispanic/Latine" designations in medical research frequently treat "Hispanic" identity as an ethnic modifier separate from a person's race. The reason for this is that a person can be "Hispanic" or "Latine" while also belonging to any race or combination of races. Nevertheless, "Hispanic/Latine" people are often referred to and studied as a single demographic category. ${ }^{56,57}$ Their health outcomes are analyzed in aggregate and compared with those of other racial groups. ${ }^{58}$ In the JAMA special edition, 3 of 4 original investigations ${ }^{59-61}$ included "Hispanic" as the only ethnic group without further disaggregation. This represents a significant limitation in health equity literature as use of this category often results in the combining of multiple, heterogeneous populations spanning dozens of countries and racial groups as well as highly variable socioeconomic, political, and cultural contexts.

For example, research on "Hispanic health" could refer to groups of impoverished Guatemalan migrant workers of largely Indigenous origins as categorically equivalent to wealthier, Whiter, Cuban US citizens of significantly more European descent. Combining these populations under the category of "Hispanic/Latine" health would at best yield limited utility in describing associated health inequities. At worst, it could hide or erase important differences in health outcomes and potential areas of contextually appropriate intervention.

To address this, we suggest the use of alternative models for understanding "Hispanic/Latine" health that recognize the immense cultural, linguistic, and racial diversity of Latin America. This can be achieved principally through the disaggregation of the "Hispanic/Latine" category by race (particularly prioritizing the identification of Black and Indigenous heritage), country of origin, immigration status, immigrant generation, class, and other key aspects to inform more critical assessments of individuals' risk of bearing systemic harms like racism, poverty, and discrimination.

Many of the limitations discussed in operationalizing race above are circumvented by direct measurement of different aspects of systemic racism. As is true for any exposure in an epidemiological study; using a proxy necessarily introduces measurement error that can introduce bias or reduce the precision in estimates of associations or causal effects. As with theory, there are readily available instruments for measuring interpersonal racism, including the Everyday Discrimination Scale (EDS), ${ }^{62}$ Measure of Indigenous Racism Experiences, ${ }^{63}$ and the Asian-American Racism-Related Stress Inventory, ${ }^{64}$ among others. In fact, a recent study used a propensity score weighting approach with the EDS to demonstrate a causal relationship between racial discrimination and depression, cardiovascular disease, and substance use disorder. ${ }^{65}$

Efforts to measure structural racism are ongoing with several different approaches currently implemented in the literature. These approaches can be broadly grouped into 3 categories, (1) measures of individual experiences of racism enacted by institutions, (2) measures that aggregate the laws, policies, and practices that comprise structural racism, and (3) measures that aggregate the downstream effects of structural racism. ${ }^{66,67}$ For example, in the third category, the state racism index, a composite measure of Black-White segregation and 
economic inequity, has been validated as a predictor of statelevel Black-White inequities in fatal police shootings. ${ }^{68}$

We advocate that observational health inequities research estimate the effect of racism by including direct measures of systemic racism. For interventional studies that seek to reduce racial health inequities there is still a role for stratification by race, however, using measures that estimate baseline exposure to systemic racism will help estimate modification effects of different interventions and determine to what degree and among which individuals an intervention is capable of mitigating the impacts of systemic racism.

\section{RECOMMENDATIONS}

\section{General Principles}

- Apply appropriate theoretical frameworks: Health inequities are never divorced from the social (between individuals and groups in a society), and structural (policies and laws that bind a society together), context of individuals' lived experiences. Therefore, it is important that studies are framed using a model of how social-structural factors might interact with the disease to produce results that are most salient for achieving health equity.

- Representation and inclusivity: One of the core tenets of critical race theory is that marginalized groups are the source of knowledge ${ }^{26}$; therefore elevating Black, Latine, and Indigenous scholars to leadership is necessary for conducting research for racial health equity. In addition to realizing the ideals of health equity, meaningful inclusion of racially marginalized scholars will facilitate innovation and enhance work quality leading to more impactful research. ${ }^{69,70}$

- Collaboration and redistribution: It is critical to draw on Black, Indigenous, Latine, and decolonial epistemologies and methodologies to inform public health research. Reimagining canon and who authoritatively crafts knowledge in public health and related fields helps realize the promises of health equity. Community consultants are experts on their own experience, so they represent an important resource for the high-quality research on their communities. Ethical practice remains paramount, and partnering with community consultants includes considering what constitutes ethical remuneration for their expertise.

\section{For Studies With New Data Collection}

- Include direct measures of systemic racism (and other relevant forms of systemic discrimination) that are appropriate for your study, rather than only using race as a proxy.

\section{Examples/Recommendations}

- Randomized clinical trial piloting referral to palliative care among patients with end-stage renal disease: include survey instrument to assess experiences of interpersonal racism among study participants to appropriately evaluate the effect of the intervention among racial group substrata.

- Quasi-experimental evaluation of the effect of mask mandates on COVID-19 case-rates: include measures of segregation at the corresponding block group or census tract level.

\section{For Studies Using Existing Secondary Data}

- If data limitations do not have granular racial and ethnic data, discuss potential biases or error introduced due to collapsing groups subject to different experiences of systemic racism (ie, Hispanic/Latine) individuals, and future study designs that might address them.

- For conditionally independent associations between race and the health outcome of interest, consider other elements of systemic racism that are not present in the study design as mediators that explain the additional effect. Discuss their impact and potential importance in future study.

- Use existing data resources such as the US Census Bureau (American Community Survey), the Centers for Disease Control and Prevention (Behavioral Risk Factor Surveillance System and National Health and Nutrition Examination Survey [NHANES]) to estimate contextual factors for inclusion in your study.

- For example, NHANES could be used to estimate statelevel food security by race and ethnicity, which may be of interest for studies evaluating cardiovascular outcomes. Though these resources are mostly national-level, there are examples of more granular data generated by advocacy groups and media collaborations that relate to health issues, such as police violence, ${ }^{71,72}$ that can be adapted for study. This also reiterates the importance of the general principles of "representation and inclusivity" and "collaboration and redistribution" and highlights the importance of cross-disciplinary and community consultant expertise.

\section{Analytic Considerations}

- Use causal diagrams ${ }^{73}$ to explicate how specific elements of systemic racism may operate in your study. Different components of racism may function as exposures, mediators, moderators (effect-measure modifiers), or confounders depending on the specific intervention of interest and their relationships to study outcome. A causal diagram will allow you to visualize your assumptions, design an analytic strategy for appropriate adjustment for bias, and put interpretation in context.

- When racism is the exposure in health or health care inequities studies, consider investigating synergism and intersectionality between racism and other societal oppressive forces such as sexism, ableism, and xenophobia.

- Avoid collapsing categories when possible (ie, combining Indigenous groups into the "Other Race" — a practice that contributes to statistical genocide).$^{74}$ If your primary analysis requires collapsing due to sample size considerations, report outcome distributions by granular race data and consider nonparametric analytic strategies that are robust to sample size for secondary analyses that avoid erasure of smaller groups. - Do not assume that White is the "default" reference population. Consider comparisons to the overall population or 
other reference groups. Provide explicit justifications for reference groups based on the study question.

\section{CONCLUSION}

Race is a proxy for exposure to systemic racism, a multilevel, historical, and ubiquitous societal force that causes adverse health outcomes among racialized persons. To move forward in achieving racial equity we must leave behind antiquated, unsubstantiated, and harmful conceptualizations of race and implement strategies that allow us to estimate the health impacts of systemic racism and ultimately, dismantle it.

\section{Read or post commentaries in response to this article.}

Key words: racism; race; inequity; disparity; intersectionality; epidemiology; community/public health

Submitted September 7, 2021; submitted, revised, November 23, 2021; accepted November 30, 2021.

Funding support: E.A. is supported by the Robert Wood Johnson Foundation Health Policy Research Scholars Program.

Acknowledgments: The authors would like to thank the Black Health Scholars Network and the E2 Social Epidemiology Laboratory for their support of this work. The authors would also like to thank Adam Mally for his assistance with figure preparation.

\section{References}

1. Cooper HLF, Fullilove M. Editorial: excessive police violence as a public health issue. J Urban Health. 2016;93(Suppl 1):1-7. 10.1007/s11524-016-0040-2

2. Webb Hooper M, Nápoles AM, Pérez-Stable EJ. COVID-19 and racial/ethnic disparities. JAMA. 2020;323(24):2466-2467. 10.1001/jama.2020.8598

3. Lee C. "Race" and "ethnicity" in biomedical research: how do scientists construct and explain differences in health? Soc Sci Med. 2009;68(6):1183-1190. 10.1016/j.socscimed.2008.12.036

4. Jones CP. Invited commentary: "race," racism, and the practice of epidemiology. Am J Epidemiol. 2001;154(4):299-304;305-306. 10.1093/aje/154.4.299

5. Chang DC, Oseni TO, Strong BL, et al. The Other Global Pandemic: Scientific Racism. Ann Surg. 2021;274(6):646-648. 10.1097/SLA.0000000000005168

6. Williams MJ, Eberhardt JL. Biological conceptions of race and the motivation to cross racial boundaries. J Pers Soc Psychol. 2008;94(6):1033-1047. 10.1037/0022-3514.94.6.1033

7. Kendi IX. Stop blaming Black people for dying of the Coronavirus. The Atlantic. Published April 14, 2020. Accessed Sep 7, 2021. https://www.theatlantic com/ideas/archive/2020/04/race-and-blame/609946/

8. Hamilton J. More questions about racial disparities in COVID-19 outcomes [All Things Considered audio]. National Public Radio. Apr 9, 2020. Accessed Sep 3, 2021. https://www.npr.org/2020/04/09/831379249/ more-questions-about-racial-disparities-in-covid-19-outcomes

9. Doubek J. Louisiana Sen. Cassidy addresses racial disparities in Coronavirus deaths. National Public Radio. Apr 7, 2020. Accessed Sep 3, 2021. https:// www.npr.org/sections/coronavirus-live-updates/2020/04/07/828827346/ louisiana-sen-cassidy-addresses-racial-disparities-in-coronavirus-deaths

10. Black health experts say surgeon general's comments reflect lack of awareness of black community. NBC News. Apr 15, 20202. Accessed Sep 3, 2021. https://www.nbcnews.com/news/nbcblk/ black-health-experts-say-surgeon-general-s-comments-reflect-lack-n1183711

11. Gabriel T. Ohio lawmaker asks racist question about Black people and handwashing. New York Times. Published Jun 11, 2020. Accessed Sep 3, 2021 https://www.nytimes.com/2020/06/11/us/politics/steve-huffman-africanamericans-coronavirus.html

12. Robinson WR, Renson A, Naimi Al. Teaching yourself about structural racism will improve your machine learning. Biostatistics. 2020;21(2):339-344. 10.1093/biostatistics/kxz040
13. Smaje C. Not just a social construct: theorising race and ethnicity. Sociology. 1997;31(2):307-327. 10.1177/0038038597031002007

14. Munyikwa M. Out from the shadows of racist anthropology. Scientific American blog. Accessed Sep 5, 2021. https://blogs.scientificamerican.com/ absolutely-maybe/out-from-the-shadows-of-racist-anthropology/

15. Tsai J, Cerdeña JP, Khazanchi R, et al. There is no 'African American Physiology': the fallacy of racial essentialism. J Intern Med. 2020;288(3):368-370. 10.1111/joim.13153

16. Gower BA, Adele Fowler L, Fernandez JR. Response to Tsai and colleagues. J Intern Med. 2020;288(3):371-372. 10.1111/joim.13152

17. Morning A. "Everyone knows it's a social construct": contemporary science and the nature of race. Sociol Focus. 2007;40(4):436-454. 10.1080/0038 0237.2007.10571319

18. Gannon M. Race Is a social construct, scientists argue. Sci American. Accessed Sep 5, 2021. https://www.scientificamerican.com/article/race-is-a-socialconstruct-scientists-argue/

19. Cerdeña JP, Plaisime MV, Tsai J. Race-conscious medicine: a response to critique. Intern Med J. 2021;51(8):1369-1370. 10.1111/imj.15460

20. Omi M, Winant H. Racial Formation in the United States. Routledge; 2014.

21. Harris Cl. Whiteness as property. Harvard Law Review. 1993;106(8):17101791. https://harvardlawreview.org/wp-content/uploads/1993/06/1707-1791_ Online.pdf

22. Link BG, Phelan J. Social conditions as fundamental causes of disease. J Health Soc Behav. 1995;35(Spec No):80-94. 10.2307/2626958

23. Krieger N. Epidemiology and the web of causation: has anyone seen the spider? Soc Sci Med. 1994;39(7):887-903. 10.1016/0277-9536(94)90202-X

24. Krieger $\mathrm{N}$. Theories for social epidemiology in the 21st century: an ecosocial perspective. Int J Epidemiol. 2001;30(4):668-677. 10.1093/ije/30.4.668

25. Krieger N. Methods for the scientific study of discrimination and health: an ecosocial approach. Am J Public Health. 2012;102(5):936-944. 10.2105/ AJPH.2011.300544

26. Ford $\mathrm{CL}$, Airhihenbuwa CO. The public health critical race methodology: praxis for antiracism research. Soc Sci Med. 2010;71(8):1390-1398. 10.1016/j. socscimed.2010.07.030

27. Bailey ZD, Krieger N, Agénor M, Graves J, Linos N, Bassett MT. Structural racism and health inequities in the USA: evidence and interventions. Lancet. 2017;389(10077):1453-1463. 10.1016/S0140-6736(17)30569-X

28. Bailey ZD, Feldman JM, Bassett MT. How structural racism works - racist policies as a root cause of U.S. racial health inequities. Malina $D$, ed. N Engl J Med. 2021;384(8):768-773. 10.1056/NEJMms2025396

29. Lawrence K, Keleher T. Structural racism. Race and Public Policy Conference; Nov 11-13, 2004; Berkeley, California.

30. Ford CL, Griffith DM, Bruce MA, Gilbert KL. Racism: Science \& Tools for the Public Health Professional. American Public Health Association; 2019.

31. Mouzon DM, McLean JS. Internalized racism and mental health among African-Americans, US-born Caribbean Blacks, and foreign-born Caribbean Blacks. Ethn Health. 2017;22(1):36-48. 10.1080/13557858.2016.1196652

32. Hoffman KM, Trawalter S, Axt JR, Oliver MN. Racial bias in pain assessment and treatment recommendations, and false beliefs about biological differences between blacks and whites. Proc Natl Acad Sci U S A. 2016;113(16): 4296-4301. 10.1073/pnas.1516047113

33. Sabin JA, Greenwald AG. The influence of implicit bias on treatment recommendations for 4 common pediatric conditions: pain, urinary tract infection, attention deficit hyperactivity disorder, and asthma. Am J Public Health. 2012; 102(5):988-995. 10.2105/AJPH.2011.300621

34. Jones CP. Levels of racism: a theoretic framework and a gardener's tale. Am J Public Health. 2000;90(8):1212-1215. 10.2105/AJPH.90.8.1212

35. Vagins DJ, McCurdy J. Cracks in the System: 20 Years of the Unjust Federal Crack Cocaine Law. American Civil Liberties Union; 2006. Accessed Sep 7, 2021. https://www.aclu.org/other/cracks-system-20-years-unjust-federalcrack-cocaine-law

36. Yehuda R, Lehrner A. Intergenerational transmission of trauma effects: putative role of epigenetic mechanisms. World Psychiatry. 2018;17(3):243-257. 10.1002/wps.20568 
37. Bower KM, Thorpe RJ Jr, Rohde C, Gaskin DJ. The intersection of neighborhood racial segregation, poverty, and urbanicity and its impact on food store availability in the United States. Prev Med. 2014;58:33-39. 10.1016/j. ypmed.2013.10.010

38. Odoms-Young A, Bruce MA. Examining the impact of structural racism on food insecurity: implications for addressing racial/ethnic disparities. Fam Community Health. 2018;41(Suppl 2 Suppl, Food Insecurity and Obesity): S3-S6. 10.1097/FCH.0000000000000183

39. Tiako MJN, McCarthy C, Meisel ZF, Elovitz MA, Burris HH, South E. Association between low urban neighborhood greenness and hypertensive disorders of pregnancy [published online ahead of print Aug 27, 2021:s-00411733786]. Am J Perinatol. 10.1055/s-0041-1733786

40. Bowleg $\mathrm{L}$. The problem with the phrase women and minorities: intersectionality-an important theoretical framework for public health. Am J Public Health. 2012;102(7):1267-1273. 10.2105/AJPH.2012.300750

41. Crenshaw K. Demarginalizing the intersection of race and sex: a black feminist critique of antidiscrimination doctrine, feminist theory and antiracist politics. Univ Chic Leg Forum. 1989;1989(1):139-167.

42. Crenshaw K. Mapping the margins: intersectionality, identity politics, and violence against women of color. Stanford Law Rev. 1991;43(6):1241. $10.2307 / 1229039$

43. Lett E, Asabor EN, Beltrán S, Dowshen N. Characterizing health inequities for the U.S. transgender Hispanic population using the Behavioral Risk Factor Surveillance System. Transgender Health. 2021;6(5):275-283. 10.1089/ trgh.2020.0095

44. Lett $\mathrm{E}$, Dowshen NL, Baker KE. Intersectionality and health inequities for gender minority Blacks in the U.S. Am J Prev Med. 2020;59(5):639-647. 10.1016/j.amepre.2020.04.013

45. Wesson P, Vittinghoff E, Turner C, Arayasirikul S, McFarland W, Wilson E. Intercategorical and intracategorical experiences of discrimination and HIV prevalence among transgender women in San Francisco, CA: a quantitative intersectionality analysis. Am J Public Health. 2021;111(3):446-456. 10.2105/ AJPH.2020.306055

46. Bauer GR. Incorporating intersectionality theory into population health research methodology: challenges and the potential to advance health equity. Soc Sci Med. 2014;110:10-17. 10.1016/j.socscimed.2014.03.022

47. Axelsson Fisk S, Mulinari S, Wemrell M, Leckie G, Perez Vicente R, Merlo J. Chronic obstructive pulmonary disease in Sweden: an intersectional multilevel analysis of individual heterogeneity and discriminatory accuracy. SSM Popul Health. 2018;4:334-346. 10.1016/j.ssmph.2018.03.005

48. Bowleg L, Bauer G. Invited reflection: quantifying intersectionality. Psychol Women Q. 2016;40(3):337-341. 10.1177/036168431665428

49. Galea S, Hernán MA. Win-Win: reconciling social epidemiology and causal inference. Am J Epidemiol. 2020;189(3):167-170. 10.1093/aje/kwz158

50. Robinson WR, Bailey ZD. Invited commentary: what social epidemiology brings to the table-reconciling social epidemiology and causal inference. Am J Epidemiol. 2020;189(3):171-174. 10.1093/aje/kwz197

51. Jackson JW, Arah OA. Invited commentary: making causal inference more social and (social) epidemiology more causal. Am J Epidemiol. 2020;189(3): 179-182. 10.1093/aje/kwz199

52. Tracy M, Cerdá M, Keyes KM. Agent-based modeling in public health: current applications and future directions. Annu Rev Public Health. 2018;39(1) 77-94. 10.1146/annurev-publhealth-040617-014317

53. Marshall BDL, Galea S. Formalizing the role of agent-based modeling in causal inference and epidemiology. Am J Epidemiol. 2015;181(2):92-99. 10.1093/aje/kwu274

54. Magaña López M, Bevans M, Wehrlen L, Yang L, Wallen GR. Discrepancies in race and ethnicity documentation: a potential barrier in identifying racial and ethnic disparities. J Racial Ethn Health Disparities. 2016;4(5):812-818. 10.1007/s40615-016-0283-3
55. Saperstein A. Double-checking the race box: examining inconsistency between survey measures of observed and self-reported race. Soc Forces. 2006;85(1):57-74. 10.1353/sof.2006.0141

56. Abraído-Lanza AF, Mendoza-Grey S, Flórez KR. A commentary on the Latin American paradox. JAMA Netw Open. 2020;3(2):e1921165. 10.1001/ jamanetworkopen.2019.21165

57. Cervantes L, Martin M, Frank MG, et al. Experiences of Latinx individuals hospitalized for COVID-19: a qualitative study. JAMA Netw Open. 2021;4(3): e210684. 10.1001/jamanetworkopen.2021.0684

58. Qeadan F, VanSant-Webb E, Tingey B, et al. Racial disparities in COVID-19 outcomes exist despite comparable Elixhauser comorbidity indices between Blacks, Hispanics, Native Americans, and Whites. Sci Rep. 2021;11(1):8738. 10.1038/s41598-021-88308-2

59. Johnston KJ, Hammond G, Meyers DJ, Joynt Maddox KE. Association of race and ethnicity and medicare program type with ambulatory care access and quality measures. JAMA. 2021;326(7):628-636. 10.1001/jama.2021.10413

60. Dieleman JL, Chen C, Crosby SW, et al. US health care spending by race and ethnicity, 2002-2016. JAMA. 2021;326(7):649-659. 10.1001/jama.2021.9937

61. Mahajan S, Caraballo C, Lu Y, et al. Trends in differences in health status and health care access and affordability by race and ethnicity in the United States, 1999-2018. JAMA. 2021;326(7):637-648. 10.1001/jama.2021.9907

62. Williams DR, Yan Yu, Jackson JS, Anderson NB. Racial differences in physical and mental health: socio-economic status, stress and discrimination. J Health Psychol. 1997;2(3):335-351. 10.1177/135910539700200305

63. Paradies YC, Cunningham J. Development and validation of the Measure of Indigenous Racism Experiences (MIRE). Int J Equity Health. 2008;7(1):9. $10.1186 / 1475-9276-7-9$

64. Liang CTH, Li LC, Kim BSK. The Asian American Racism-Related Stress Inventory: development, factor analysis, reliability, and validity. J Couns Psychol. 2004;51(1):103-114. 10.1037/0022-0167.51.1.103

65. Chen S, Mallory AB. The effect of racial discrimination on mental and physical health: a propensity score weighting approach. Soc Sci Med. 2021;285: 114308. 10.1016/j.socscimed.2021.114308

66. Groos M, Wallace M, Hardeman R, Theall KP. Measuring inequity: a systematic review of methods used to quantify structural racism. J Health Disp Res Pract. 2018;11(2):190-206. https://digitalscholarship.unlv.edu/jhdrp/vol11/ iss $2 / 13 /$

67. Agénor M, Perkins $C$, Stamoulis $C$, et al. Developing a database of structural racism-related state laws for health equity research and practice in the United States. Public Health Rep. 2021;136(4):428-440. 10.1177/00333549 20984168

68. Mesic A, Franklin L, Cansever A, et al. The relationship between structural racism and black-white disparities in fatal police shootings at the state level. J Natl Med Assoc. 2018;110(2):106-116. 10.1016/j.jnma.2017.12.002

69. Hofstra B, Kulkarni VV, Munoz-Najar Galvez S, He B, Jurafsky D, McFarland DA. The diversity-innovation paradox in science. Proc Natl Acad Sci U S A. 2020;117(17):9284-9291. 10.1073/pnas.1915378117

70. Hewlett SA, Marshall M, Sherbin L. How diversity can drive innovation. Harv Bus Rev. 2013. https://hbr.org/2013/12/how-diversity-can-drive-innovation

71. An introduction to the citizens police data project. Invisible Institute. Accessed Sep 6, 2021. https://invisible.institute/police-data

72. Mapping police violence. Accessed Aug 8, 2021. https://mappingpolice violence.org/

73. Greenland S, Pearl J, Robins JM. Causal diagrams for epidemiologic research. Epidemiology. 1999;10(1):37-48.

74. Huyser KR, Horse AJY, Kuhlemeier AA, Huyser MR. COVID-19 Pandemic and indigenous representation in public health data. Am J Public Health. 2021; 111(S3):S208-S214. 10.2105/AJPH.2021.306415 